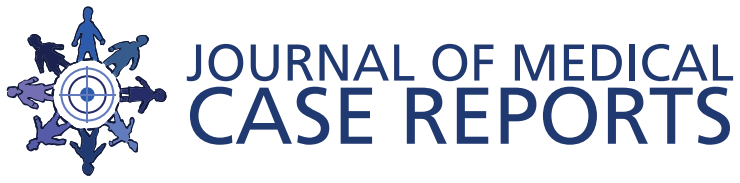

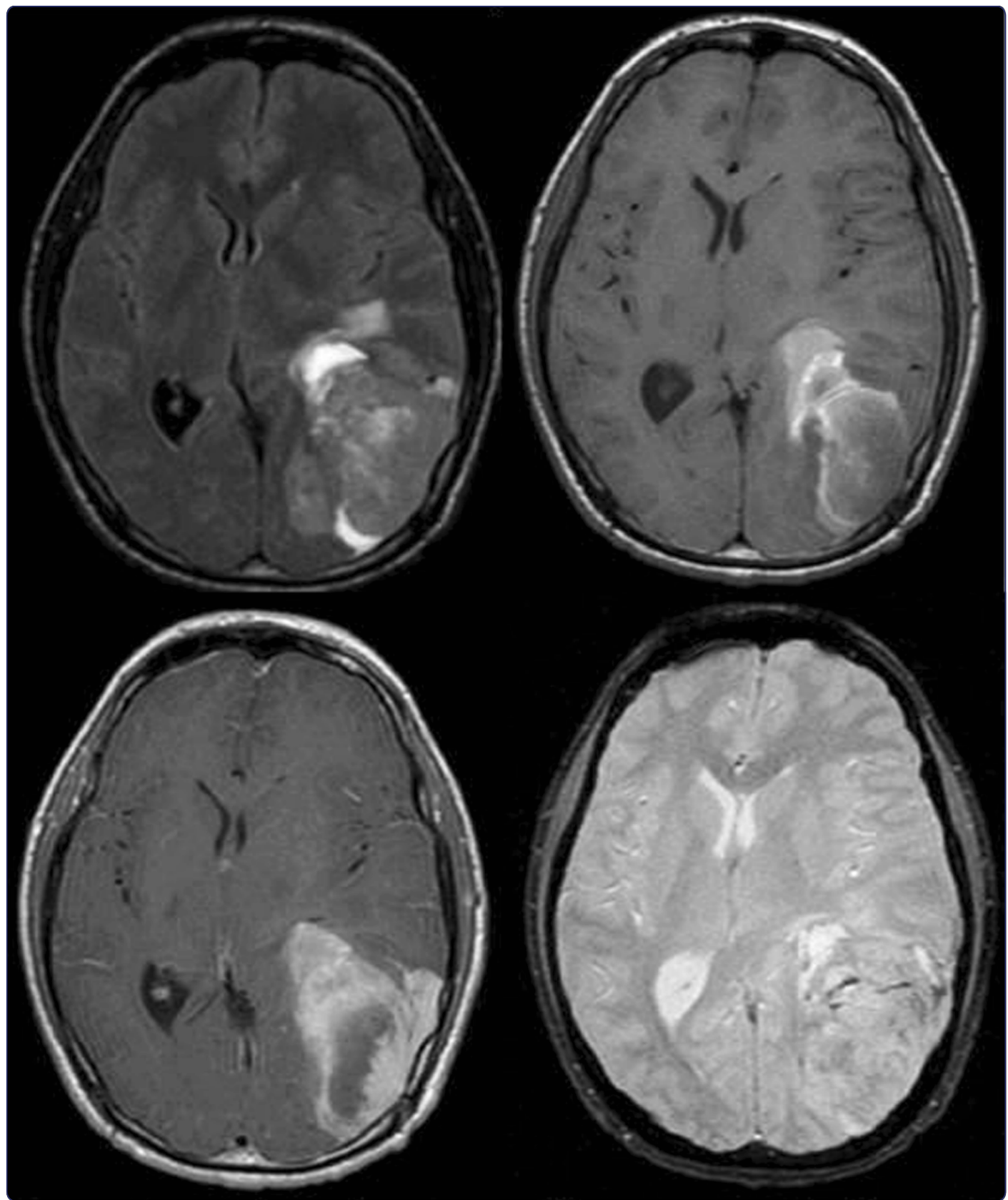

Supratentorial primitive neuroectodermal tumor in an adult: a case report and review of the literature

Lawandy et al. 


\title{
Supratentorial primitive neuroectodermal tumor in an adult: a case report and review of the literature
}

Shokry Lawandy ${ }^{1,2}$, Omid R Hariri ${ }^{1,2^{*}}$, Dan E Miulli ${ }^{1,2}$, Jenny Amin², Tanya Minasian ${ }^{1,2}$, Ravi K Gupta ${ }^{3}$ and Javed Siddiqi ${ }^{1,2}$

\begin{abstract}
Introduction: Supratentorial primitive neuroectodermal tumors predominantly occur in children, and are rare in the adult population. Less than 100 cases of supratentorial primitive neuroectodermal tumor have been reported in adults internationally. Our case study reports this rare incident.

Case presentation: A 22-year-old Hispanic man presented with headaches, blurry vision, diplopia, intermittent vomiting, and grossly decreased vision. A magnetic resonance image showed a left posterior parietal heterogeneously enhancing mass measuring $4.2 \mathrm{~cm} \times 7.2 \mathrm{~cm} \times 7.0 \mathrm{~cm}$. After craniotomy for resection and decompression, the mass was histologically revealed to be a supratentorial primitive neuroectodermal tumor. Standardized immunohistochemical studies for this mass were carried out.

Conclusion: We have concluded that immunohistochemical and genetic workup should be included in the standardized pathological workup for primitive neuroectodermal tumors in order to provide more prognostic information. Based on our current literature review, we propose an immunohistochemical panel.
\end{abstract}

\section{Introduction}

Supratentorial primitive neuroectodermal tumors (sPNETs) are known to be tumors of the pediatric population. According to the World Health Organization, sPNETs are described as a cerebral or suprasellar embryonal grade IV tumor made up of undifferentiated or poorly differentiated neuro-epithelial cells which have the capacity for, or display, divergent differentiation along neuronal, astrocytic, ependymal, muscular or melanocytic lines. Approximately $1 \%$ of pediatric brain tumors are sPNETs. However, they can sporadically occur in adults. To date, only less than 100 sPNET cases have been reported in adult patients [1].

In an extensive case review series by Ohba et al., it was determined that the mean age of diagnosis in adults was 35.2 years of age, with one peak between the ages of

\footnotetext{
* Correspondence: ohaririucla@gmail.com

${ }^{1}$ Department of Neurosurgery, Arrowhead Regional Medical Center, 400

North Pepper Ave, Colton, CA 92324, USA

${ }^{2}$ Department of Surgery, College of Osteopathic Medicine, Western University of Health Sciences, 309 E. 2nd St, Pomona, CA 91766, USA Full list of author information is available at the end of the article
}

the second and third decades. Throughout all age groups, more males were prone to be diagnosed with pNETS than females [1].

Of the PNETs in the central nervous system (CNS) $5.6 \%$ are supratentorial. Moreover, the locations of these tumors are almost equally distributed in the frontal, temporal, and parietal lobes [1]. Less than $50 \%$ of these patients have a survival rate of 5 years post-diagnosis [2]. Radiographically, the best diagnostic clue is the presence of a large, complex hemispheric mass with minimal peritumor vasogenic edema. Cerebral hemispheric PNETs have a mean diameter of approximately $5 \mathrm{~cm}$ at diagnosis. PNETs often appear with necrosis, intra-tumor hemorrhage, cysts, and calcification (50-70\%). On computed tomography, these lesions appear as isodense or hyperdense. On magnetic resonance imaging (MRI), these tumors appear as well-delimited, inhomogeneous, and variably contrast-enhanced lesions. Furthermore, on T1weighted images, PNETs appear hypointense, and on T2weighted images they appear hyperintense [3-6].

Histologically, the highly cellular tumor consists of anaplastic cells with small round to oval hyperchromatic 
nuclei surrounded by scanty cytoplasm (Figure 1). After a review of the literature, we propose a novel pathological and genetic panel workup for brain masses in adults that are suspected to be PNETs based on radiographic studies and intra-operative pathological diagnosis.

\section{Case presentation}

A 22-year-old Hispanic man was referred to a neurosurgery clinic by his ophthalmologist, who was concerned about increased intracranial pressure after noticing papilledema on fundoscopic examination. The patient had been complaining of headaches, blurry vision, diplopia, and intermittent vomiting for a month. He reported that his symptoms had been worsening over the past week. The patient has no other significant past medical history and has been in general good health besides the recent onset of headaches. He was a resident of California. Furthermore, on physical examination, left horizontal nystagmus and grossly decreased vision on the right temporal and right nasal hemifield were noticed.

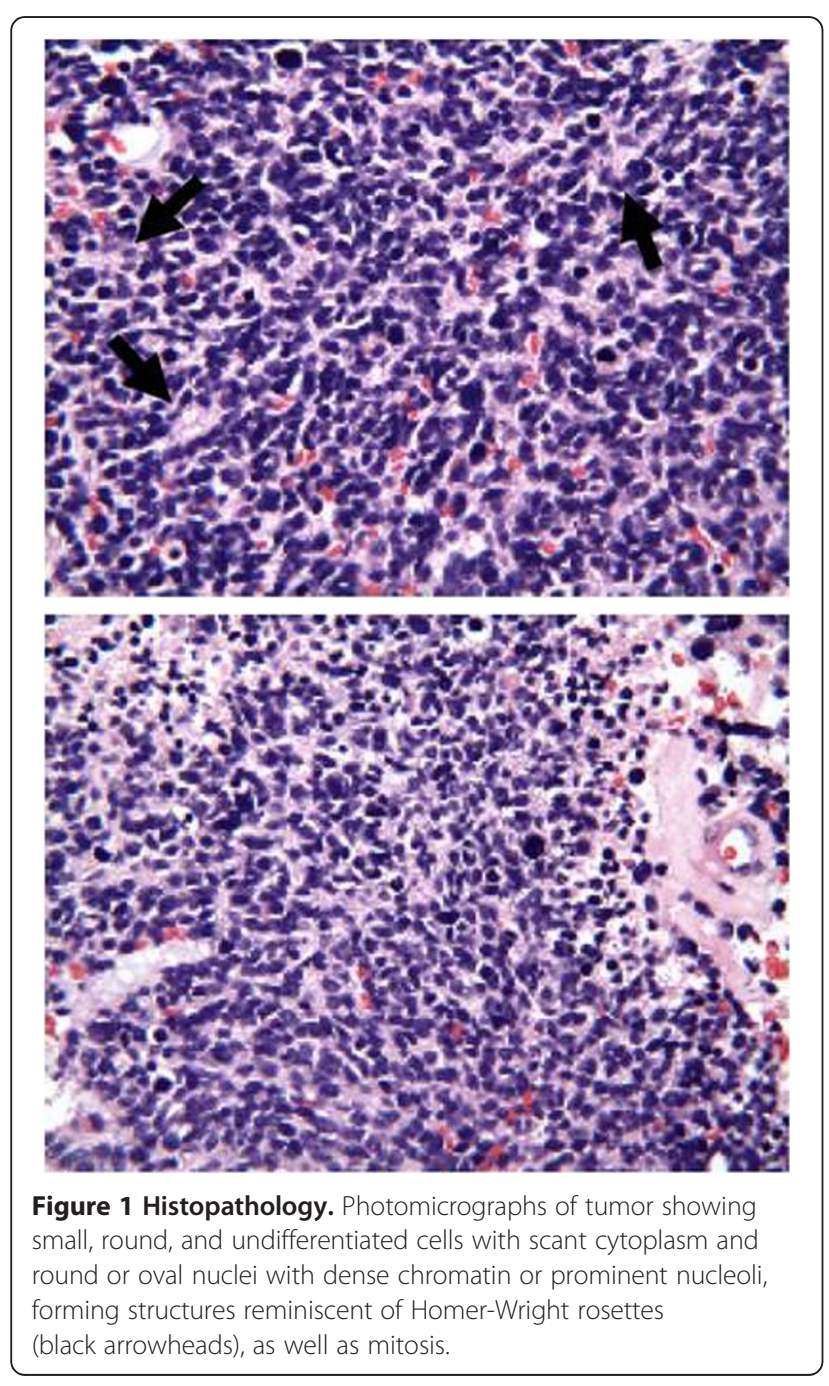

A brain MRI revealed a left posterior parietal, irregular, hemorrhagic mass measuring approximately $4.2 \mathrm{~cm} \times$ $7.2 \mathrm{~cm} \times 7.0 \mathrm{~cm}$, enhancing heterogeneously, and associated surrounding vasogenic edema and a $8.7 \mathrm{~mm}$ midline shift from left to right. Compressions of the atrium of the left lateral ventricle, and the third ventricle, as well as some effacement of the quadrigeminal plate cistern, particularly on the left were noted (Figure 2).

The patient underwent a left parieto-occipital craniotomy with resection, and the ultimate goal of debulking of mass. Intra-operatively, the lesion was noted to have a necrotic center. Initially, a fresh frozen specimen showed the tumor to be glioblastoma multiforme. However, a studied permanent specimen indicated a primitive neuroectodermal tumor with 5 to 10 mitoses per high-power field. There were many cells immunoreactive to neuronspecific enolase, CD56, and small cell neuroendocrine carcinoma. However, none were immune-reactive to synaptophysin or glial fibrillary acidic protein. There were no intra-operative or postoperative complications.

\section{Discussion}

Therapeutic approaches are close to non-existent because the pathological mechanisms underlying sPNETs are poorly understood. Some of the proposed genetic alterations include: isochromosome 17q; losses of chromosomes

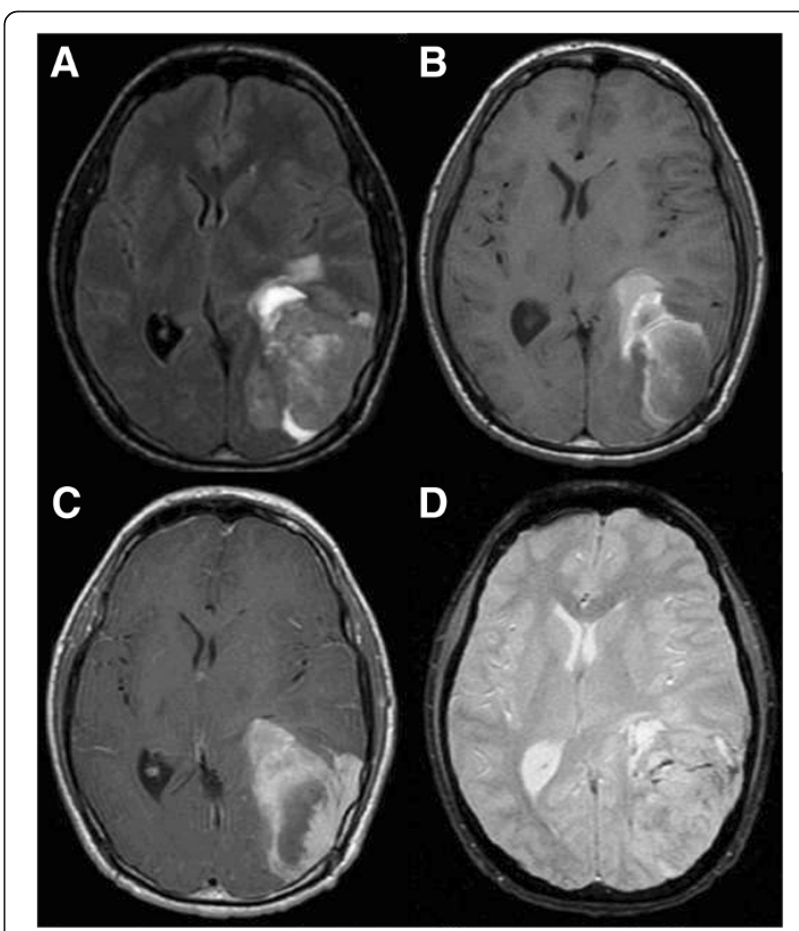

Figure 2 Magnetic resonance image of mass. A: Flair showing mild peritumoral edema, $\mathbf{B}$ : T1-weighted image without contrast, C T1-weighted image with contrast showing homogenous enhancement, D: Gradient echo. 
$6 \mathrm{q}, 9 \mathrm{q}, 10 \mathrm{q}, 11$, and 16q; trisomy-1q; and mutations of TP53. PNETs are classified into peripheral and central. Intracranial peripheral PNET has a better prognosis in comparison to central PNET. Accordingly, it is essential to differentiate between the two types. Chromosomal translocation of chromosome $(11 ; 22)$ is phenomenal in that it is unique to central and not peripheral PNETs. Thus, the usage of immunohistochemical assay for CD99 and fluorescence in situ hybridization (FISH) assay for the $(11 ; 22)$ translocation have granted the capability to distinguish between intracranial peripheral and intracranial central PNET $[1,7]$. More importantly, in a study by Hayden et al., it has been suggested that mutations in $I D H 1$, which encodes for cytoplasmic isocitrate dehydrogenase, are among the most frequent mutations in SPNET, and are much more prevalent in adult cases in comparison to pediatric cases [8]. Furthermore, Gessi et al. have shown that a high incidence of TP53 mutations and the absence of amplification of the $c-m y c / N-m y c$ genes in adult SPNET, in comparison to pediatric PNET, suggest that adult sPNET should be an independent subset of tumors among CNS-PNETs [3].

Even though no prospective treatment studies have been conducted to date, and no optimal treatment regimen for sPNET has been established, a complete surgical excision followed by radiation therapy has been proved to be crucial in many retrospective studies [9-12]. Radiotherapy to the entire neuroaxis is recommended because cerebrospinal dissemination at the time of diagnosis was found in almost $10 \%$ of all cases.

\section{Conclusion}

An extensive review of the current literature suggests that immunohistochemical and genetic assays are becoming essential markers for prognosis and possible treatment of sPNETs. As we discover more about all the mentioned studies, it will become essential to integrate them into our standard pathological workup for brain masses in adults that are highly suspicious to be PNETs based on radiographic studies and preliminary intra-operative pathological diagnosis.

We propose immunohistochemical and genetic assays panels, which to the best of our knowledge has not been proposed in the literature before, and we have decided to use this panel in our institutions. We believe that initially a genetic assay should evaluate any potential mutations to IDH1 (more prevalent in adult cases) to differentiate adult sPNET from other possible pediatric PNETs. Furthermore, an evaluation of TP53 mutations (high incidents in adults) and $c-m y c / N-m y c$ genes (absence of amplification in adults) should be evaluated in order to differentiate between a true sPNET and any pediatric PNETs. Subsequently, a CD99 and a FISH assay for the $(11 ; 22)$ translocation (phenomenal in that it is unique to central and not peripheral PNETs) should be conducted in order to distinguish between intracranial peripheral and intracranial central PNETs, because the prognosis of central and peripheral PNETs are drastically different, as mentioned previously.

\section{Consent}

Written informed consent was obtained from the patient for publication of this case report and accompanying images. A copy of the written consent is available for review by the Editor-in-Chief of this journal.

\section{Competing interests}

The authors declare that they have no competing interests.

\section{Authors' contributions}

$\mathrm{SL}$ was the physician for the patient in the manuscript and was a contributor in writing the manuscript. $\mathrm{OH}$ was a major contributor in writing the molecular neurobiology segments of the manuscript, as well as the main editor. DM was the chief editor of this manuscript. JA and TM have also contributed to the various editorial comments and to preparing the manuscript, and histological examination. JS contributed to the surgical segment of the manuscript and was the main attending neurosurgeon for this case. RG contributed the neuro-radiology included with the manuscript. All authors read and approved the final manuscript.

\section{Author details}

'Department of Neurosurgery, Arrowhead Regional Medical Center, 400 North Pepper Ave, Colton, CA 92324, USA. ²Department of Surgery, College of Osteopathic Medicine, Western University of Health Sciences, 309 E. 2nd St, Pomona, CA 91766, USA. ³ Department of Radiology, Harbor-UCLA Medical Center, 1000 West Carson Street, PO Box 2910, Torrance, CA 90509, USA.

Received: 11 June 2012 Accepted: 19 September 2012 Published: 24 October 2012

\section{References}

1. Ohba S, Yoshida K, Hirose Y, Ikeda E, Kawase T: A supratentorial primitive neuroectodermal tumor in an adult: a case report and review of the literature. J Neuro-Oncol 2008, 86(2):217-224. Epub 2007/08/24.

2. Pizer BL, Weston $C L$, Robinson KJ, Ellison DW, Ironside J, Saran F, et al: Analysis of patients with supratentorial primitive neuro-ectodermal tumours entered into the SIOP/UKCCSG PNET 3 study. Eur J Cancer 2006, 42(8):1120-1128. Epub 2006/04/25.

3. Gessi M, Setty P, Bisceglia M, Zur Muehlen A, Lauriola L, Waha A, et al: Supratentorial primitive neuroectodermal tumors of the central nervous system in adults: molecular and histopathologic analysis of 12 cases. Am J Surg Pathol 2011, 35(4):573-582. Epub 2011/03/08.

4. Pickuth $D$, Leutloff U: Computed tomography and magnetic resonance imaging findings in primitive neuroectodermal tumours in adults. Br J Radiol 1996, 69(817):1-5. Epub 1996/01/01.

5. Pigott TJ, Punt JA, Lowe JS, Henderson MJ, Beck A, Gray T: The clinical, radiological and histopathological features of cerebral primitive neuroectodermal tumours. Br J Neurosurg 1990, 4(4):287-297. Epub 1990/01/01.

6. Shingu T, Kagawa T, Kimura Y, Takada D, Moritake K, Hoshii Y: Supratentorial primitive neuroectodermal tumor in an aged patient-case report. Neurol Med Chir 2005, 45(10):530-535. Epub 2005/10/26.

7. Gyure KA, Prayson RA, Estes ML: Extracerebellar primitive neuroectodermal tumors: A clinicopathologic study with bcl-2 and CD99 immunohistochemistry. Ann Diagn Pathol 1999, 3(5):276-280. Epub 1999/11/11.

8. Hayden JT, Fruhwald MC, Hasselblatt M, Ellison DW, Bailey S, Clifford SC: Frequent $I D H 1$ mutations in supratentorial primitive neuroectodermal tumors (sPNET) of adults but not children. Cell Cycle 2009, 8(11):1806-1807. Epub 2009/05/05. 
9. Chan AW, Tarbell NJ, Black PM, Louis DN, Frosch MP, Ancukiewicz M, et al: Adult medulloblastoma: prognostic factors and patterns of relapse. Neurosurgery 2000, 47(3):623-631. Discussion 31-2. Epub 2000/09/12.

10. Frost PJ, Laperriere NJ, Wong CS, Milosevic MF, Simpson WJ, Pintilie M: Medulloblastoma in adults. Int I Radiat Oncol Biol Phys 1995, 32(4):951-957. Epub 1995/07/15.

11. Krampulz T, Hans VH, Oppel F, Dietrich U, Puchner MJ: Long-term relapsefree survival with supratentorial primitive neuroectodermal tumor in an adult: a case report. J Neuro-Oncol 2006, 77(3):291-294. Epub 2006/03/11.

12. Mikaeloff $Y$, Raquin MA, Lellouch-Tubiana A, Terrier-Lacombe MJ, Zerah M, Bulteau C, et al: Primitive cerebral neuroectodermal tumors excluding medulloblastomas: a retrospective study of 30 cases. Pediatr Neurosurg 1998, 29(4):170-177. Epub 1999/01/07.

doi:10.1186/1752-1947-6-36

Cite this article as: Lawandy et al: Supratentorial primitive neuroectodermal tumor in an adult: a case report and review of the literature. Journal of Medical Case Reports 2012 6:361.

\section{Submit your next manuscript to BioMed Central and take full advantage of:}

- Convenient online submission

- Thorough peer review

- No space constraints or color figure charges

- Immediate publication on acceptance

- Inclusion in PubMed, CAS, Scopus and Google Scholar

- Research which is freely available for redistribution 